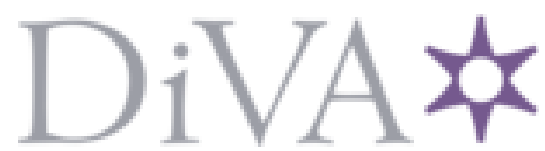

http://www.diva-portal.org

\title{
Preprint
}

This is the submitted version of a paper presented at 2016 IEEE Innovative Smart Grid Technologies Asia (ISGT-Asia).

Citation for the original published paper:

Pham, C-T., Månsson, D. (2016)

On the development of an equivalent electrical circuit model for energy storages for smart grid applications.

In: Innovative Smart Grid Technologies - Asia (ISGT-Asia), 2016 IEEE https://doi.org/10.1109/ISGT-Asia.2016.7796364

N.B. When citing this work, cite the original published paper.

Permanent link to this version:

http://urn.kb.se/resolve?urn=urn:nbn:se:kth:diva-199602 


\section{On the development of an equivalent electrical circuit model for energy storages for smart grid applications}

\author{
Cong-Toan Pham \\ School of Electrical Engineering \\ KTH Royal Institute of Technology \\ Stockholm, Sweden \\ ctpham@kth.se
}

\author{
Daniel Månsson \\ School of Electrical Engineering \\ KTH Royal Institute of Technology \\ Stockholm, Sweden \\ manssond@kth.se
}

\begin{abstract}
Energy storages are indispensable in regard to the development of the smart grid with increasing fluctuating power generation. A valid choice of energy storages is important to ensure the efficiency of the system and selected application. This study describes an initial approach to model energy storage systems as an equivalent circuit. This model is capable of analyzing different load scenarios and simulating the storage's behavior in dependence of three circuit components, i.e., resistance, inductance and capacitance. These model parameters define the storage system and can be used to test existing storage devices or find an optimal setting for selected load conditions. The model is tested in a load scenario with a residential building supported by photovoltaic generation.
\end{abstract}

Keywords-energy storage; smart grid; simulation; physical system modelling; photovoltaic; battery; flywheel

\section{INTRODUCTION}

Energy storage systems (ESS) partake in the development of the smart grid, especially in respect to the growing use of renewable energy sources (RES), e.g., wind and solar power. Various storage technologies are integrated in different load situations (on \& off grid) on most power system levels (generation, distribution and consumption), with a varying implementation depth, to effectively balance the energy demand and supply. Current research often focuses on the applicability, benefits and optimizations of selected ESS in, for example, wind power integration, power quality, load management etc. [1-3]. Each ESS is unique and can provide services for the purposes it was designed for. E.g., batteries (BESS) might be more flexible in their scope of application compared to pumped hydro stations (PHS) due to the slow response times of PHS. New variations of energy storage media are constantly under development, but their value in practice is yet to be determined $[4,5]$. This implies that there is a general interest in finding and choosing the "optimal" energy storage for certain applications. The choice of ESS has often been investigated from the viewpoint of the storage technology itself, whether its physical properties match with the requirements of the application it should be used for [6]. A reverse approach is to question what type and combination of ESS would fit for a specific load configuration.
One of the main objectives of this study is to emphasize the analogy between physical systems and propose an initial approach to incorporate those in a single model. The general energy storage model is then applied to different load and power production scenarios. The needed type and capacity of energy storages can be deduced based on these parameters. This study analyzes a household application powered through a $3.4 \mathrm{~kW}$ peak $\left(\mathrm{kW}_{\mathrm{p}}\right)$ photovoltaic $(\mathrm{PV})$ system.

\section{Methodology}

\section{Analogy between physical systems}

Energy is the capability of a system to perform work and exists in several forms (e.g. potential, electric, chemical etc.) whose physical principles are employed in the existing types of storages. Although each type of energy storage is governed by different physical laws, a common denominator can be found, allowing us to describe their dynamic behavior in a unified model. This unifying concept is energy [7].

All physical systems are related through power and energy by the product of their system variables. Reference [7] gives the two interacting system variables as effort $(e)$ and flow $(f)$. Effort represents the "intensive" property (e.g., voltage, force, pressure etc.) whereas flow depicts the "extensive" property (e.g., current, velocity, flow rate etc.).

$$
\begin{aligned}
& \text { power }=e f \\
& \text { energy }=\int_{t_{0}}^{t_{1}} e f d t
\end{aligned}
$$

The relationship between these two variables differs between each physical system characterized by lumped system elements. These system elements describe the properties of the physical systems, e.g., the electrical resistance quantifies the measure of electric current passing through a conductor. In an electrical system resistance, inductance and capacitance are defined as the system elements which are equivalent to friction, mass and spring constant in a mechanical one. The analogy of the system elements is reflected in Table 1. 
TABLE I. ANALOGY BETWEEN PHYSICAL QUANTITIES (LINEAR COMPONENTS)

\begin{tabular}{|c|c|c|c|c|c|c|c|c|}
\hline & \multicolumn{2}{|c|}{ Electrical system } & \multicolumn{2}{|c|}{ Mechanical system (rotational) } & \multicolumn{2}{|c|}{ Fluidic system } & \multicolumn{2}{|c|}{ Thermal system } \\
\hline $\begin{array}{c}\text { System } \\
\text { variables }\end{array}$ & Voltage: $U$ & Current: $I$ & Axial torque: $\tau$ & $\begin{array}{c}\text { Angular } \\
\text { velocity: } \omega\end{array}$ & $\begin{array}{c}\text { Fluid } \\
\text { pressure: } P\end{array}$ & $\begin{array}{l}\text { Fluid flow } \\
\text { rate: } Q\end{array}$ & $\begin{array}{c}\text { Temperature: } \\
T \\
\end{array}$ & $\begin{array}{l}\text { Heat flow } \\
\text { rate: } q\end{array}$ \\
\hline \multirow{3}{*}{$\begin{array}{c}\text { System } \\
\text { parameters }\end{array}$} & \multicolumn{2}{|l|}{ Resistance: } & \multicolumn{2}{|c|}{ Rotational damper: $\quad B$} & \multicolumn{2}{|c|}{ Fluid resistance: } & \multicolumn{2}{|c|}{ Thermal Resistance: $R_{t}$} \\
\hline & \multicolumn{2}{|l|}{ Capacitance: } & \multicolumn{2}{|c|}{ Torsional stiffness: $k$} & \multicolumn{2}{|l|}{$\begin{array}{l}\text { Fluid reservoir: } \\
\& \text { pressure tank }\end{array}$} & \multicolumn{2}{|l|}{ Specific heat: } \\
\hline & \multicolumn{2}{|l|}{ Inductance: } & \multicolumn{2}{|c|}{ Moment of inertia: $\quad I_{m}$} & \multicolumn{2}{|c|}{ Inertance: $\quad L_{f}$} & \multicolumn{2}{|c|}{$\begin{array}{l}\text { Heat coefficient: } \quad C_{h}, C_{r} \\
\text { (Convection \& radiation) }\end{array}$} \\
\hline \multirow{3}{*}{$\begin{array}{l}\text { Governing } \\
\text { relations }\end{array}$} & \multicolumn{2}{|c|}{$U=R I$} & \multicolumn{2}{|c|}{$\tau=B \omega$} & \multicolumn{2}{|c|}{$P=R_{f} Q$} & \multicolumn{2}{|c|}{$q=\frac{1}{R_{t}} T, q=C_{h} A T, q=C_{r} T^{4}$} \\
\hline & \multicolumn{2}{|c|}{$U=\frac{1}{C} \int I d t$} & \multicolumn{2}{|c|}{$\tau=k \int \omega d t$} & \multicolumn{2}{|c|}{$P=\frac{1}{C_{f}} \int Q d t$} & \multicolumn{2}{|c|}{-} \\
\hline & \multicolumn{2}{|c|}{$U=L \frac{d I}{d t}$} & \multicolumn{2}{|c|}{$\tau=I_{m} \frac{d \omega}{d t}$} & \multicolumn{2}{|c|}{$P=L_{f} \frac{d Q}{d t}$} & \multicolumn{2}{|c|}{$q=m C_{p} \frac{d T}{d t}$} \\
\hline
\end{tabular}

Due to the above shown analogies we propose an equivalent electrical circuit model representative for all other energy storage types. Resistance $R$ reflects the dissipation of energy; an equivalent to losses through friction and heat losses. The capacitance $C$ and the inductance $L$ translate to, for a mechanical system, the inverse of a spring constant and the moment of inertia, respectively. Each lumped element comprises of further geometrical and material parameters, e.g., resistance is dependent on length, resistivity and crosssectional area while fluidic friction relates to fluid viscosity and flow turbulence [7]. In this work we will continue to refer to the system components as electrical elements.

\section{Energy storage model}

The dynamic behavior of an ESS is reproduced by the intrinsic parameters $R, L$ and $C$, but different systems may not be able to satisfactorily store or deliver the power from a highly fluctuating source/load (wind, solar). The model presented here is limited to only the central storage unit itself, thus, considering ancillary equipment as ideal power transmitters. E.g., modelling a flywheel (FESS) would usually require an additional, separate model for the motor/generator unit as well. Fig. 1 illustrates the scheme of the storage model coupled with the power profiles. In this paper, the model approach will be shown for batteries (potential energy storage) and flywheels (kinetic energy storage). Other ESS can similarly be modelled (e.g., compressed air energy storage (CAES), superconducting energy storage (SMES), PHS etc.). In the following sections we will show that the storage types can be described as nonlinear ordinary differential equations (ODE).

\subsection{Potential energy - battery model (BESS)}

Batteries currently receive great attention and progress, especially in the area in electric vehicles (EV), but also in the advancement of the smart grid. Lithium-ion based batteries have emerged as the currently leading technology in terms of efficiency, cyclic life and energy density and they are continuously investigated to maximize their benefits [1]. Alternative technologies are catching up through newly developed design methods and materials [8,9]. Great effort has been invested to implement batteries in various applications and several models have been introduced to simulate real storage behavior via equivalent circuit elements [10-12]. These results can directly be applied by us.

A commonly used model evolved from Electrochemical Impedance Spectroscopy which describes the electrochemical processes through traditional circuit elements $(R, L$ and $C)$. The equivalent circuit model, in the simplest form (see Fig. 1a) [13], consists of a voltage source $U_{b}$ (the open circuit voltage of the battery), a series resistance $R$ (the series ohmic drop through contact and material resistances) and a parallel RCbranch $\left(R_{c t}, C_{D}\right)$ (the charge transfer resistance and the pseudocapacitance originating from mass transfer limitations). An additional parallel resistance can be added to specify a selfdischarge process. BESS types differ by those parameters, e.g., the values for lead-acid batteries were identified in [11] to be approximately $R=0.06 \Omega, R_{c t}=0.07 \Omega$ and $C_{D}=205 \mathrm{~F}$ compared to a Li-ion battery with $R=0.01 \Omega, R_{c t}=0.02 \Omega$ and $C_{D}=1700 \mathrm{~F}$. Note, the values can vary between batteries of the same BESS dependent on the method used, but the difference sufficiently distinguishes between different types. In reality, the circuit parameters also display non-linearity through charge and discharge or degradation over time which can be captured by having time dependent parameters [12]. This study however does not consider this non-linearity but rather focuses on the applicability of ESS in smart grid applications where constant parameters are believed to be sufficient. Degradation effects will be included in future works.

The battery model follows a similar approach to the theory of Ragone plots [14] regarding a power source $(P)$ instead of a commonly used voltage source. The original equation is derived from Kirchhoff's voltage law. The power developed in the source is related to the voltage $U(t)$ of the source times the current $I(t)$ through it, replacing the voltage source with $U(t)=P(t) / I(t)$

$$
\frac{P(t)}{I(t)}=R I(t)+U_{C d}(t)+U_{b}
$$

Inserting the expressions for the capacitive and resistive components, described above in (3), and solving for the voltage over the parallel RC-branch returns (4). 


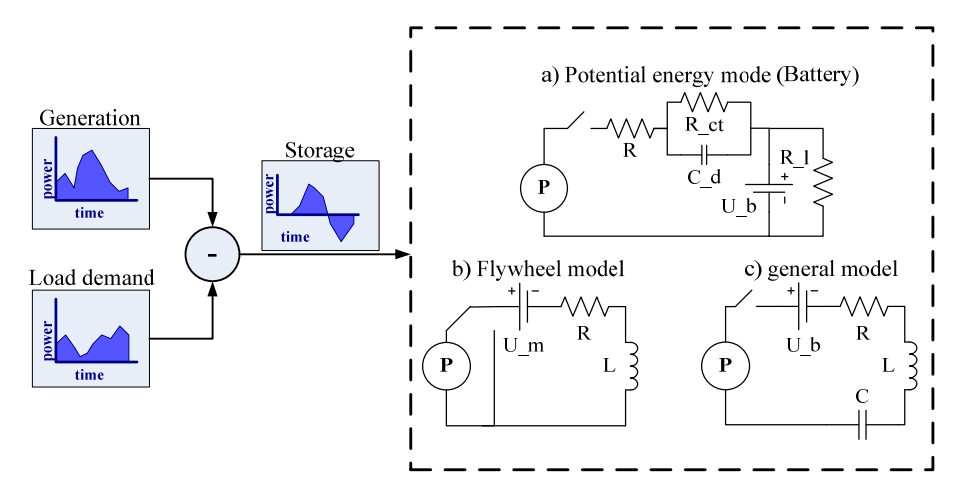

Figure 1: Equivalent circuit models for two different energy storages and general model. The difference between the generation and load demand serves as the power in- and output into the storage model. a) potential energy storage model (battery), b) kinetic energy storage model (flywheels with magnetic bearings), c) general energy storage model (without self-discharge).

$$
\frac{P(t)}{I(t)}=R\left(C_{D} \frac{d U_{C d}(t)}{d t}+\frac{U_{C d}(t)}{R_{c t}}\right)+U_{C d}(t)+U_{b}
$$

Multiplying with $I=I_{c t}+I_{c}$, and rearranging the terms, results in a non-linear ODE with a quadratic derivative of $U_{C d}$.

$$
\begin{aligned}
& 0=\left(\frac{d U_{C d}(t)}{d t}\right)^{2}+\frac{\left(R_{c t}+2 R\right) U_{C d}(t)+R_{c t} U_{b}}{R C_{D} R_{c t}}\left(\frac{d U_{C d}(t)}{d t}\right) \ldots \\
& \ldots+\frac{\left(R_{c t}+R\right)\left(U_{C d}(t)\right)^{2}+R_{c t} U_{b} U_{C d}(t)-R_{c t}{ }^{2} P(t)}{R C_{D}{ }^{2} R_{c t}{ }^{2}}
\end{aligned}
$$

After some algebraic steps we achieve (6).

$$
\begin{aligned}
& \frac{d U_{C d}(t)}{d t}=-\frac{\left(R_{c t}+2 R\right) U_{C d}(t)+R_{c t} U_{b}}{2 R C_{D} R_{c t}} \ldots \\
& \ldots+\sqrt{\frac{\left(\frac{\left(R_{c t}+2 R\right) U_{C d}(t)+R_{c t} U_{b}}{2 R C_{D} R_{c t}}\right)^{2}-}{\frac{\left(R_{c t}+R\right)\left(U_{C d}(t)\right)^{2}+R_{c t} U_{b} U_{C d}(t)-R_{c t}{ }^{2} P(t)}{R C_{D} R_{c t}{ }^{2}}}}
\end{aligned}
$$

This non-linear ODE is then numerically solved through Matlab using the ode45 solver [15]. With $U_{C d}$ known the other system currents and voltages can easily be computed. We now can also include leakage losses $R_{l}$ in parallel to $U_{b}$.

$$
I_{b a t t}(t)=I(t)-\frac{U_{b}}{R_{l}}
$$

Identifying the equivalent leakage resistance $R_{l}$ is difficult and would require in-depth investigation. Self-discharge processes are complex including for example chemical and mechanical electrode corrosion, internal losses due to partial electron movements in the electrolyte etc. $[14,16]$. Instead of detailed mathematical description of self-discharge processes a more modest approach is suitable for our application. Measurement and observation allow us to monitor self-discharge in terms of lost energy capacity over time. If the self-discharge rate $(S D)$ for a specific period $(\Delta t)$ of a Li-Ion battery is known, then dependent on its maximum capacity $\left(Q_{0}\right)$ the equivalent linear loss relates to (8) by using the relation $P=U^{2} / R$ and
$P \Delta t=W=Q U$. The energy drop is the difference between the initial and end capacity $\triangle W=W_{0^{-}} W_{l}=U\left(Q_{0^{-}}(1-S D) Q_{0}\right)=S D Q_{0} U$.

$$
R_{l}=\frac{U_{b}^{2} \Delta t}{P \Delta t}=\frac{U_{b} \Delta t}{S D Q_{0}}
$$

The total current into the battery determines the power input. The stored energy is the time integral of the power.

$$
\begin{gathered}
P_{\text {store }}(t)=I_{\text {batt }} U_{b}=\left(C_{D} \frac{d U_{C d}(t)}{d t}+\frac{U_{C d}(t)}{R_{c t}}-\frac{U_{b}}{R_{l}}\right) U_{b}(9) \\
W_{\text {store }}(t)=\int P_{\text {store }}(t) d t
\end{gathered}
$$

\subsection{Kinetic energy - flywheel model (FESS)}

Flywheels have gained popularity especially in the vehicle industry as ancillary short term energy storages for uninterruptable power supply or power quality. Recently FESS also became attractive for large-scale applications in the Smart Grid providing up to $20 \mathrm{MW}$ [1]. Considerable work has been invested to optimize flywheel design to improve their selfdischarge characteristics. Newly developed designs mostly involve vacuum operation and magnetic bearings to reduce friction losses $[1,17,18]$.

A modest way of simulating a FESS is a RL-circuit [19], illustrated in Fig. 1c. It consists of a series resistance reflecting the internal losses of the system due to friction by bearings and drag. Inductance $L$ compares with the moment of inertia $I_{m}$ defined by the shape and material properties of the rotor itself. The main limitation is the critical angular velocity which depends on the system design. Velocities near this threshold expose the system to instability, higher losses and even damage. Specifying the losses depends on various conditions summarized in [20]. The moment of inertia varies with the shape of the rotating material and is in general described as the volume integral over the mass in respect to the distance from the rotation axis. For a cylindrical shell of mass $m$, radius $r$ and with negligible shell thickness the expressions results in (11) [21]. Alternatively, if the geometries are not available one can deduce $I_{m}$ by relating it to the tensile strength $\sigma$ of the material, the maximum operation speed $\omega_{\max }$, the mass $m$ and material density $\rho$ [20]. With $\sigma=\rho r^{2} \omega_{\max }^{2}$ solved for $r$ we obtain (11). 


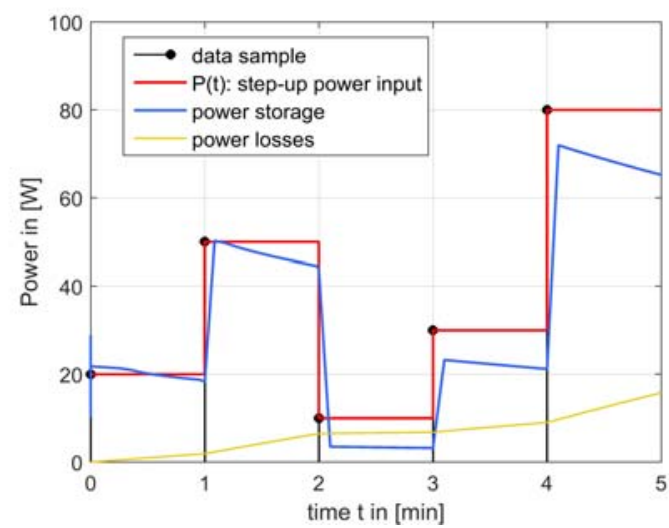

Figure 2: Example of power distribution in storage model during charging process.

$$
L \equiv I_{m}=m r^{2}=\frac{\sigma}{\omega_{\max }^{2}} \frac{m}{\rho}
$$

The kinetic energy stored is then determined by the shape factor $K$, the moment of inertia $I_{m}$ and the angular velocity $\omega$ [19].

$$
W_{\text {store }}(t)=K I_{m} \omega^{2}(t)
$$

The system variables of this mechanical system are torque $\tau$ and angular velocity $\omega$. The governing relation for the acceleration of a rotational mass is $\tau=I_{m} d \omega / d t$. The losses in a vacuum operated flywheel can be simplified to the linear expression $\tau=B \omega$ with losses mainly caused by the used bearing types, i.e., for magnetic bearings the drag torque is low and can be approximated by the following expression valid for a horizontal rotor with $b$ the number of poles in the induction machine [21]. In vertical systems the torque losses are even less.

$$
\tau_{b, \text { magnet }}(t)=m g\left(3.2 \times 10^{-5}+1.3 \times 10^{-8} b \omega\right)
$$

The loss term is split into a constant torque sink (which can be modelled as a voltage source) and the equivalent resistance $R \equiv B$ of $1.3 \times 10^{-8} b$. For ball bearings the loss term differs and requires much more information on the flywheel design [21]. Viscous drag is vanishingly low for vacuum systems. Applying the KVL on Fig. 1c yields the following expression.

$$
\frac{P(t)}{I(t)}=R I(t)+L \frac{d I(t)}{d t}+U_{m}
$$

Separating the variables gives then (15).

$$
\frac{d I(t)}{d t}=\frac{P(t)}{L I(t)}-\frac{R}{L} I(t)-\frac{U_{m}}{L}
$$

Solving for the current $I$, the voltage over the inductance is then $U_{l}=L \cdot d I / d t$. Other power losses due to compressor, seals, control system etc. could also be included, but for now we focus on the main components directly connected to the central storage unit itself.

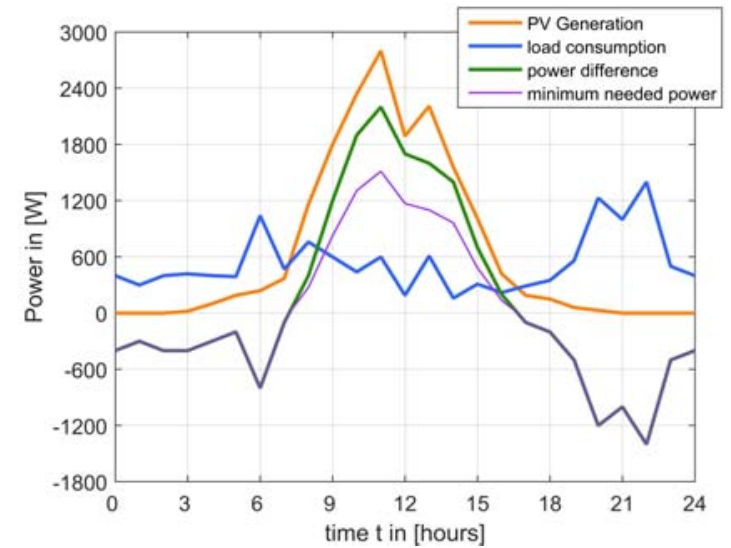

Figure 3: generation and load profile of a household with $3.4 \mathrm{~kW}_{\mathrm{p}}$ photovoltaic system during a summer day.

\subsection{General energy storage}

Other ESS as Supercapacitors, PHS, SMES and CAES can be modelled in the same fashion as the storages presented so far. The only difference lies in the combination of the resistive, capacitive and inductive elements in the model (Fig. 1d).

$$
\frac{P(t)}{I(t)}=L C \frac{d^{2} U_{C}(t)}{d t^{2}}+R C \frac{d U_{C}(t)}{d t}+U_{C}(t)+U_{b}
$$

Loss terms have to be included in (15) for the specific ESS type accordingly. Hybrid systems can also be analyzed by this modelling approach (e.g. battery + capacitor, PHS + FESS etc.) and are currently under investigation.

\subsection{Modelling fluctuating load conditions}

The second objective of this work is to analyze the capability of ESS under fluctuating supply and load conditions. To generate any load scenario, the input into the ESS is determined by the power profile of the user. With available generation and load profiles, the difference between both regulates the requirements on the power inserted or drawn from the ESS. Importantly, the input data is rarely given as a continuous function but rather as discrete information in various sampling rates (seconds, hours etc.).

Developing an approximate continuous function to feed into the power function $P(t)$ in each of the storage expressions (3), (14) and (16) is cumbersome and analytically difficult to solve. We therefore suggest a more direct method by using the sampled data. We assume the power input as a step-up function $\left(P(t)=P\left(t_{j}\right)=\right.$ const. $)$ in each time-step $\Delta t$. Depending on the time-constant the storage does or does not manage to follow the input power in the given time-step. For the consecutive time-steps the end values of the previous time-steps are used as initial conditions $y_{0}$ (see Fig. 2). $y_{j}$ stands for the general solution of the ODEs $\left(U_{c}, I\right.$ etc. $)$ at the time $t_{j}$. The accuracy of the model directly relates to the sample rate of the data used. Smaller time-steps increase the accuracy but also the computation time.

$$
y_{j+1,0}=y_{j}\left(t_{j}+\Delta t\right) \quad j=1 \ldots n
$$



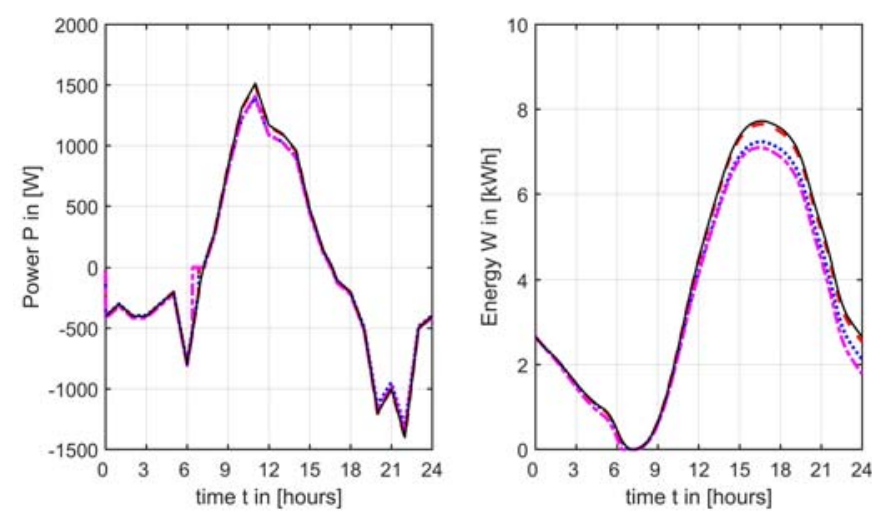

Figure 4: Comparison between lead-acid, Li-Ion and NiMH batteries for a PVsupported household. Lead-acid (blue dotted): $\mathrm{R}=0.06 \Omega, \mathrm{R}_{\mathrm{ct}}=0.07 \Omega$ $\mathrm{C}_{\mathrm{D}}=205 \mathrm{~F}, \mathrm{U}_{\mathrm{b}}=6 \mathrm{~V}, \mathrm{SD}=0.06, \mathrm{q}_{0}=13 \mathrm{Ah}$. Li-Ion (red dashed): $\mathrm{R}=0.01 \Omega$, $\mathrm{R}_{\mathrm{ct}}=0.02 \Omega, \mathrm{C}_{\mathrm{D}}=1700 \mathrm{~F}, \mathrm{U}_{\mathrm{b}}=11.1 \mathrm{~V}, \mathrm{SD}=0.03, \mathrm{q}_{0}=13 \mathrm{Ah}$. NiMH (violet mixed): $\mathrm{R}=0.06 \Omega, \mathrm{R}_{\mathrm{ct}}=0.08 \Omega, \mathrm{C}_{\mathrm{D}}=503 \mathrm{~F}, \mathrm{U}_{\mathrm{b}}=7.2 \mathrm{~V}, \mathrm{SD}=0.3, \mathrm{q}_{0}=5 \mathrm{Ah}$;

\section{RESULTS AND DISCUSSION}

In this section the proposed storage model will be tested on a household application supported by a $3.4 \mathrm{~kW}_{\mathrm{p}}$ photovoltaic system. Generation and demand power curves are presented in Fig. 3. The orange curve shows the provided solar power and the blue curve represents the power consumption of the household. The power difference between generation and load demand (green curve) determines the required power in- and output of the ESS model. For an optimal case the energy storage should absorb and deliver just the amount given by the minimum required power (purple curve). Deviations between the actual stored energy and the optimal situation provide us the information whether the storage used is suitable or not. In this paper the described storage models for batteries and flywheels are tested for this application.

\subsection{Battery application}

The type of BESS is defined, see above, by the parameters $R, R_{c t}, C_{D}$ and $U_{b}$. Three different types of batteries (Lead-acid, Li-Ion, NiMH) are exemplarily assessed for this application [11] (Fig. 4). All battery types feature satisfying dynamic behavior for the PV-application. They are able to follow the ideal power reference curve and only slightly differ through their losses. Li-Ion displays the least losses while charging and discharging compared to lead-acid and NiMH. Due to the short period of only a day the self-discharge losses for $\mathrm{NiMH}$ are not clearly observable, but would have greater influence over several cycles.

It is usually not feasible to dimension the energy storage to cover the complete energy demand. In this case however, it is interesting to deduce the theoretical capacity needed for the daily consumption. With the charge capacity $Q_{0}$ for each battery, shown in Fig. 4, the number of stacked cells can be estimated. For an ideal $7.7 \mathrm{kWh}$ storage capacity about 100 stacked cells of this lead-acid, 140 of this Li-Ion and 215 of this NiMH would be required.
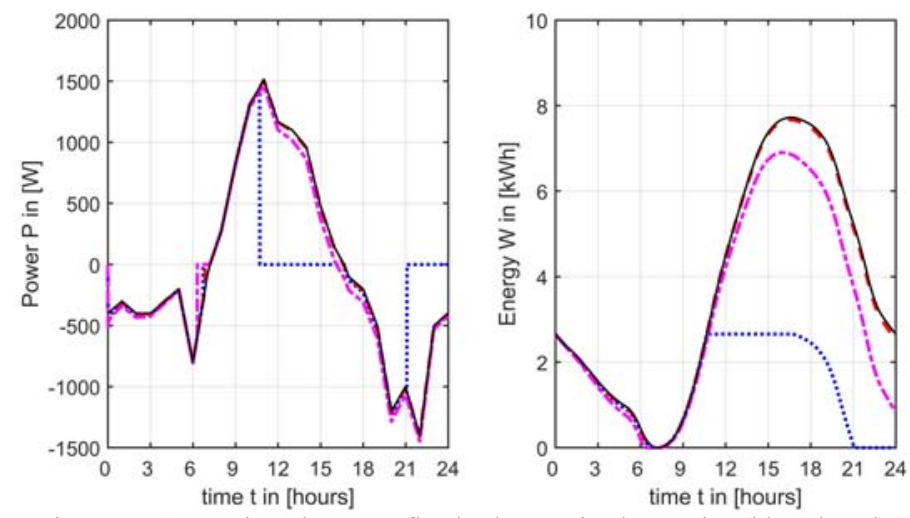

Figure 5: Comparison between flywheels: 1. Fixed capacity (blue dotted) $\mathrm{L}=1.4 \mathrm{H}, \mathrm{R}=7.8 \mathrm{e}^{-8} \Omega, \mathrm{W}_{\max }=2.66 \mathrm{kWh} ; 2$. Theoretical solution (red dashed) $\mathrm{L}=8.4 \mathrm{H}, \mathrm{R}=7.8 \mathrm{e}^{-8} \Omega ; 3$. Effect of high resistance values (violet mixed) $\mathrm{L}=1.4 \mathrm{H}, \mathrm{R}=0.001 \Omega$.

\subsection{Flywheel application}

Flywheels feature high power density but low capacity. The limit in FESS is set by the critical angular velocity. Two examples for FESS are presented: a vacuum operated flywheel (Carbon epoxy (40\%)) with magnetic bearing, fixed moment of inertia $I_{m}$ and finite capacity and one with found $I_{m}$ with speed restraints $(\sim 2600 \mathrm{rad} / \mathrm{s})$ (Fig. 5): $L=I_{m}=1.4 \mathrm{kgm}^{2}, R=7.8$ $10^{8} \mathrm{Ohm}, \quad \mathrm{K}=0.5, \quad \sigma=1.2 \mathrm{GPa}, \quad \rho=1580 \mathrm{~kg} / \mathrm{m}^{3}$. For this household application FESS with the required capacity do exist. With low $R$ values the inertia of the system is negligible small for this rather slow application. To store $7.7 \mathrm{kWh}$ a stack of 6 flywheels with each $1.4 \mathrm{kgm}^{2}$ running on $2600 \mathrm{rad} / \mathrm{s}$ is required.

Increasing $I_{m}$ of the system too much is not recommended as the energy increases linearly with the mass but also increases as $\omega^{2}$ (see equation (12)). Comparable products for 1-3 $\mathrm{kWh}$ applications range between $I_{m}=30-100 \mathrm{kgm}^{2}$ with lower operation speeds around $800 \mathrm{rad} / \mathrm{s}$ [22]. If the resistance is higher even in the milliohm range the losses increases significantly which is why reducing friction losses in flywheels is essential. Therefore, the losses of the different ESS will be investigated in future work.

\section{CONCLUSION}

The results show that the capability of storage systems to adapt to load changes is dependent on the intrinsic parameters and their relation to each other. Different physical systems share a common concept of energy, enabling a description of the dynamic behavior through a unified model approach. This model can analyze different load situations on ESS and provides information on their suitability for the selected application. This allows us to test specific ESS types and products based on system parameters. 
This model is expandable by increasing the complexity of the model using basic components $(R, L$ and $C$ ) to describe ESS more accurately. Furthermore, the advantage lies in predicting the capacity of a needed storage device for a selected application. We can also formulate constraints to limit the effort or flow value to find optimal parameters to fulfill the demand. With the optimized parameters we can further deduce geometrical and material properties of the storage in question.

Further investigation is necessary to strengthen the results through analytical or empirical methods. This approach only considers linear elements, but real energy storages also exhibit non-linear characteristics which will be included in future work. The next step will also involve hybrid systems.

\section{REFERENCES}

[1] X. Lou, J.Wang, M. Dooner, J. Clarke, "Overview of current development in electrical energy storage technologies and the application potential in power system operation," Applied Energy, vol. 137, pp. 511-536, 2015.

[2] R. Sebastian, R. Pena-Alzola, "Control and simulation of flywheel energy storage for a wind diesel power system," Electrical Power and Energy Systems, vol. 64, pp. 1049-1056, 2015.

[3] C. Abbey, G. Joos, "Energy Management Strategies for Optimization of Energy Storage in Wind Power Hybrid System," in Power Electronics Specialists Conference, 2005. PESC '05. IEEE 36th, pp. 2066-2072.

[4] Daniel Månsson, "On the suitability of Using Halbach Arrays as Potential Energy Storage Media," Progress In Electromagnetics Research B, vol. 58, pp. 151-166, 2014.

[5] Daniel Månsson, "On the Optimization of Halbach Arrays as Energy Storage Media”, Progress in Electromagnetics Research B, 2015, Vol. 62, pp. 277-288.

[6] C.T.Pham, D. Månsson, "Suitability analysis of Fuzzy Logic as an evaluation method for the selection of energy storage technologies in Smart Grid applications," International Symposium on Smart Electric Distribution Systems and Technologies (EDST), Vienna 8 Sep. 2015, IEEE Conference Publications, pp. 452-457

[7] Peter E. Wellstead, "Introduction to Physical System Modelling," Control Systems Principles, [ONLINE] Available at: http://www.control-systems-principles.co.uk. [Accessed 22 April 2016].
[8] X. Zhang,Y. Tang, F. Zhang, C. Lee, "A Novel Aluminium-Graphite Dual-Ion Battery," Advanced Energy Materials, 2016

[9] H. Lee, S. Choi, "An origami paper-based bacteria-powered battery," Nano Energy, 2015, Vol. 15, pp. 549-557.

[10] H. Rahimi-Eichi, M. Chow, "Adaptive Parameter Identification and State-of-Charge Estimation of Lithium-Ion Batteries," IECON 2012 $38^{\text {th }}$ Annual Conference on IEEE Industrial Electronics Society, Montreal Oct. 2012, pp. 4012-4017.

[11] T. Hu, B. Zanchi, J. Zhao, "Determining battery parameters by simple algebraic method," Proceedings of the 2011 American Control Conference, 2011, pp. 3090-3095.

[12] J. Brand, Z. Zhang, R. K. Agarwal, "Extraction of battery parameters of the equivalent circuit model using a multi-objective generic algorithm," Journal of Power Sources, 2014, Vol. 204, pp. 729-737.

[13] D. Linden, T. B. Reddy, Linden's Handbook of Batteries, $4^{\text {th }}$ ed, McGraw-Hill Education, 2011, Chapter 6.1ff

[14] T. Christen, M. W. Carlen, "Theory of Ragone plots," Journel of Power Sources, 2000, Vol. 91, pp. 210-216.

[15] Mathworks Documentation, "Choose an ODE solver", [ONLINE] Available at: http://se.mathworks.com/help/matlab/math/choose-an-odesolver.html, [Accessed 10 May 2016].

[16] M. Swierczynski, D. Stroe, A. Stan, R. Teodorescu, S. K. Kær, "Investigation on the Self-discharge of the $\mathrm{LiFePO}_{4} / \mathrm{C}$ Nanophosphate Battery Chemistry at Different Conditions," Transportation Electrification Asia-Pacific (ITEC Asia-Pacific), 2014 IEEE Conference and Expo, Beijing Aug. 2014, pp. 1-6.

[17] S. Mukoyama et al., "Development of REBCO HTS Magnet of Magnetic Bearing for Large Capacity Flywheel Energy Storage System," $27^{\text {th }}$ International Symposium on Superconductivity, ISS 2014, 2014, Physics Procedia, Vol. 65, pp. 253-256.

[18] J. Abrahamsson, "Kinetic Energy Storage and Magnetic Bearings for Vehicular Applications," Dissertation at Uppsala University. 2014.

[19] A. Ter-Gazarian, "Energy Storage for Power Systems," $2^{\text {nd }}$ ed, IET, 2011.

[20] R. A. Serway, J. W. Jewett, "Physics for Scientists and Engineers," $6^{\text {th }}$ ed., Thomson Brooks/Cole, 2004.

[21] G. Genta, Kinetic Energy Storage - Theory and Practice of Advanced Flywheel Systems, $1^{\text {st }}$ ed, Elsevier, 1985, pp. 169-245.

[22] Active Power, "Products," [ONLINE] Available at: http://www.activepower.com/de/products/, [Accessed 10 May 2016]. 